\title{
CROSS-LINGUAL TRANSFER OF SENTIMENT CLASSIFIERS
}

\author{
Marko ROBNIK-ŠIKONJA
}

Faculty of Computer and Information Science, University of Ljubljana

\section{Kristjan REBA}

Faculty of Computer and Information Science, University of Ljubljana

\section{Igor MOZETIČ}

Jožef Stefan Institute

Robnik-Šikonja, M., Reba, K., Mozetič, I. (2021): Cross-lingual transfer of sentiment classifiers. Slovenščina 2.o, 9(1): 1-25.

DOI: https://doi.org/10.4312/slo2.0.2021.1.1-25

Word embeddings represent words in a numeric space so that semantic relations between words are represented as distances and directions in the vector space. Cross-lingual word embeddings transform vector spaces of different languages so that similar words are aligned. This is done by mapping one language's vector space to the vector space of another language or by construction of a joint vector space for multiple languages. Cross-lingual embeddings can be used to transfer machine learning models between languages, thereby compensating for insufficient data in less-resourced languages. We use cross-lingual word embeddings to transfer machine learning prediction models for Twitter sentiment between 13 languages. We focus on two transfer mechanisms that recently show superior transfer performance. The first mechanism uses the trained models whose input is the joint numerical space for many languages as implemented in the LASER library. The second mechanism uses large pretrained multilingual BERT language models. Our experiments show that the transfer of models between similar languages is sensible, even with no target language data. The performance of cross-lingual models obtained with the multilingual BERT and LASER library is comparable, and the differences are language-dependent. The transfer with CroSloEngual BERT, pretrained on only three languages, is superior on these and some closely related languages.

Keywords: natural language processing, machine learning, text embeddings, sentiment analysis, BERT models 


\section{INTRODUCTION}

Word embeddings are representations of words in numerical form, as vectors of typically several hundred dimensions. The vectors are used as input to machine learning models; for complex language processing tasks, these generally are deep neural networks. The embedding vectors are obtained from specialised neural network-based embedding algorithms, e.g., fastText (Bojanowski et al., 2017) for morphologically-rich languages. Word embedding spaces exhibit similar structures across languages, even when considering distant language pairs like English and Vietnamese (Mikolov et al., 2013). This means that embeddings independently produced from monolingual text resources can be aligned, resulting in a common cross-lingual representation, called cross-lingual embeddings, which allows for fast and effective integration of information in different languages.

There exist several approaches to cross-lingual embeddings. The first group of approaches uses monolingual embeddings with an optional help from a bilingual dictionary to align the pairs of embeddings (Artetxe et al., 2018a). The second group of approaches uses bilingually aligned (comparable or even parallel) corpora to construct joint embeddings (Artetxe and Schwenk, 2019). This approach is implemented in the LASER library ${ }^{1}$ and is available for 93 languages. The third type of approaches is based on large pretrained multilingual masked language models such as BERT (Devlin et al., 2019). In this work, we focus on the second and third group of approaches. In particular, from the third group, we apply two variants of BERT models, the original multilingual BERT model (mBERT), trained on 104 languages, and trilingual CroSloEngual BERT (Ulčar and Robnik-Šikonja, 2020) trained on Croatian, Slovene, and English (CSE BERT).

Sentiment annotation is a costly and lengthy operation, with a relatively low inter-annotator agreement (Mozetič et al., 2016). Large annotated sentiment datasets are, therefore, rare, especially for low-resourced languages. The transfer of already trained models or datasets from other languages would increase the ability to study sentiment-related phenomena for many more languages than possible today.

1 https://github.com/facebookresearch/LASER 
Our study aims to analyse the abilities of modern cross-lingual approaches for the transfer of trained models between languages. We study two cross-lingual transfer technologies, using a joint vector space computed from parallel corpora with the LASER library and multilingual BERT models. The advantage of our study is sizeable comparable classification datasets in 13 different languages, which gives credibility and general validity to our findings. Further, due to the datasets' size, we can reliably test different transfer modes: direct transfer between languages (called a zero-shot transfer) and transfer with enough fine-tuning data in the target language. In the experiments, we study two cross-lingual transfer modes based on projections of sentences into a joint vector space. The first mode transfers trained models from source to target languages. A model is trained on the source language(s) and used for classification in the target language(s). This model transfer is possible because texts in all processed languages are embedded into the common vector space. The second mode expands the training set with instances from other languages, and then all instances are mapped into the common vector space during neural network training. Besides the cross-lingual transfer, we analyse the quality of representations for the Twitter sentiment classification and compare the common vector space for several languages constructed by the LASER library, multilingual BERT models, and the traditional bag-of-words approach. The results show a relatively low decrease in predictive performance when transferring trained sentiment prediction models between similar languages and superior performance of multilingual BERT models covering only three languages.

The paper is divided into four more sections. In Section 2, we present background on different types of cross-lingual embeddings: alignment of monolingual embeddings, building a common explicit vector space for several languages, and large pretrained multilingual contextual models. We also discuss related work on Twitter sentiment analysis and cross-lingual transfer of classification models. In Section 3, we present a large collection of tweets from 13 languages used in our empirical evaluation, the implementation details of our deep neural network prediction models, and the evaluation metrics used. Section 4 contains four series of experiments. We first evaluate different representation spaces and compare the LASER common vector space with 
multilingual BERT models and convential bag-of-ngrams. We then analyse the transfer of trained models between languages from the same language group and from a different language group, followed by expanding datasets with instances from other languages. In Section 5, we summarise the results and present ideas for further work.

\section{BACKGROUND AND RELATED WORK}

Word embeddings represent each word in a language as a vector in a high dimensional vector space so that the relations between words in a language are reflected in their corresponding embeddings. Cross-lingual embeddings attempt to map words represented as vectors from one vector space to another so that the vectors representing words with the same meaning in both languages are as close as possible. Søgaard et al. (2019) present a detailed overview and classification of cross-lingual methods.

Cross-lingual approaches can be sorted into three groups, described in the following three subsections. The first group of methods uses monolingual embeddings with (an optional) help from bilingual dictionaries to align the embeddings. The second group of approaches uses bilingually aligned (comparable or even parallel) corpora for joint construction of embeddings in all handled languages. The third type of approaches is based on large pretrained multilingual masked language models such as BERT (Devlin et al., 2019). In contrast to the first two types of approaches, the multilingual BERT models are typically used as starting models, which are fine-tuned for a particular task without explicitly extracting embedding vectors.

In Section 2.1, we first present background information on the alignment of individual monolingual embeddings. We describe the projections of many languages into a joint vector space in Section 2.2, and in Section 2.3, we present variants of multilingual BERT models. In Section 2.4, we describe related work on Twitter sentiment classification. Finally, in Section 2.5, we outline the related work on cross-lingual transfer of classification models.

\subsection{Alignment of monolingual embeddings}

Cross-lingual alignment methods take precomputed word embeddings for each language and align them with the optional use of bilingual dictionaries. 
Two types of monolingual embedding alignment methods exist. The first type of approaches map vectors representing words in one of the languages into the vector space of the other language (and vice-versa). The second type of approaches maps embeddings from both languages into a joint vector space. The goal of both types of alignments is the same: the embeddings for words with the same meaning must be as close as possible in the final vector space. A comprehensive summary of existing approaches can be found in (Artetxe et al., 2018a). The open-source vecmap ${ }^{2}$ library contains implementations of methods described in (Artetxe et al., 2018a), and can align monolingual embeddings using a supervised, semi-supervised, or unsupervised approach.

The supervised approach requires the use of a bilingual dictionary, which is used to match embeddings of equivalent words. The embeddings are aligned using the Moore-Penrose pseudo-inverse, which minimises the sum of squared Euclidean distances. The algorithm always converges but can be caught in a local maximum. Several methods (e.g., stochastic dictionary introduction or frequency-based vocabulary cut-off) are used to help the algorithm climb out of local maxima. A more detailed description of the algorithm is given in (Artetxe et al., 2018b).

The semi-supervised approach uses a small initial seeding dictionary, while the unsupervised approach is run without any bilingual information. The latter uses similarity matrices of both embeddings to build an initial dictionary. This initial dictionary is usually of low but sufficient quality for later processing. After the initial dictionary (either by seeding dictionary or using similarity matrices) is built, an iterative algorithm is applied. The algorithm first computes optimal mapping using the pseudo-inverse approach for the given initial dictionary. The optimal dictionary for the given embeddings is then computed, and the procedure iterates with the new dictionary.

When constructing mappings between embedding spaces, a bilingual dictionary can help as its entries are used as anchors for the alignment map for supervised and semi-supervised approaches. However, lately, researchers have proposed methods that do not require a bilingual dictionary but rely on the 
adversarial approach (Conneau et al., 2018) or use the words' frequencies (Artetxe et al., 2018b) to find a required transformation. These are called unsupervised approaches.

\subsection{Projecting into a joint vector space}

To construct a common vector space for all the processed languages, one requires a large aligned bilingual or multilingual parallel corpus. The constructed embeddings must map the same words in different languages as close as possible in the common vector space. The availability and quality of alignments in the training set corpus may present an obstacle. While Wikipedia, subtitles, and translation memories are good sources of aligned texts for large languages, less-resourced languages are not well-presented and building embeddings for such languages is a challenge.

LASER (Language-Agnostic SEntence Representations) is a Facebook research project focusing on joint sentence representation for many languages (Artetxe and Schwenk, 2019). Strictly speaking, LASER is not a word but sentence embedding method. Similarly to machine translation architectures, LASER uses an encoder-decoder architecture. The encoder is trained on a large parallel corpus, translating a sentence in any language or script to a parallel sentence in either English or Spanish (whichever exists in the parallel corpus), thereby forming a joint representation of entire sentences in many languages in a shared vector space. The project focused on scaling to many languages; currently, the encoder supports 93 different languages. Using LASER, one can train a classifier on data from just one language and use it on any language supported by LASER. A vector representation in the joint embedding space can be transformed back into a sentence using a decoder for the specific language.

\subsection{Multilingual BERT and CroSloEngual BERT}

BERT (Bidirectional Encoder Representations from Transformers) embedding (Devlin et al., 2019) generalises the idea of a language model (LM) to masked LMs, inspired by the cloze test, which checks understanding of a text by removing a few words, which the participant is asked to replace. The masked LM randomly masks some of the tokens from the input, and 
the task is to predict the missing token based on its neighbourhood. BERT uses transformer neural networks (Vaswani et al., 2017) in a bidirectional sense and further introduces the task of predicting whether two sentences appear in a sequence. The input representation of BERT are sequences of tokens representing sub-word units. The input is constructed by summing the embeddings of corresponding tokens, segments, and positions. Some widespread words are kept as single tokens; others are split into sub-words (e.g., frequent stems, prefixes, suffixes-if needed down to single letter tokens). The original BERT project offers pre-trained English, Chinese, and multilingual model. The latter, called mBERT, is trained on 104 languages simultaneously.

To use BERT in classification tasks only requires adding connections between its last hidden layer and new neurons corresponding to the number of classes in the intended task. The fine-tuning process is applied to the whole network, and all the parameters of BERT and new class-specific weights are fine-tuned jointly to maximise the log-probability of correct labels.

Recently, a new type of multilingual BERT models emerged that reduce the number of languages in multilingual models. For example, CSE BERT (Ulčar and Robnik-Šikonja, 2020) uses Croatian, Slovene (two similar less-resourced languages from the same language family), and English. The main reasons for this choice are to represent each language better and keep sensible sub-word vocabulary, as shown by Virtanen et al. (2019). This model is built with the cross-lingual transfer of prediction models in mind. As CSE BERT includes English, we expect that it will enable a better transfer of existing prediction models from English to Croatian and Slovene.

\subsection{Twitter sentiment classification}

We present a brief overview of the related work on automated sentiment classification of Twitter posts. We summarise the published labelled sets used for training the classification models and the machine learning methods applied for training. Most of the related work is limited to only English texts.

To train a sentiment classifier, one needs a reasonably large training dataset of tweets already labelled with the sentiment. One can rely on a proxy, e.g., 
emoticons used in the tweets, to determine the intended sentiment; however, high-quality labelling requires the engagement of human annotators. There exist several publicly available and manually labelled Twitter datasets. They vary in the number of examples from several hundred to several thousand, but to the best of our knowledge, so far, none exceeds 20,000 entries. Saif et al. (2013) describe eight Twitter sentiment datasets and introduce a new one that contains separate sentiment labels for tweets and entities. Rosenthal et al. (2015) provide statistics for several of the 2013-2015 SemEval datasets.

There are several supervised machine learning algorithms suitable to train sentiment classifiers from sentiment labelled tweets. For example, in the SemEval-2015 competition, before the rise of deep neural networks, the most often used algorithms for the sentiment analysis on Twitter (Rosenthal et al., 2015) were support vector machines (SVM), maximum entropy, conditional random fields, and linear regression. In other cases, frequently used classifiers were naive Bayes, k-nearest neighbours, and even decision trees. Often, SVM was shown as the best performing classifier for the Twitter sentiment. However, only recently, when researchers started to apply deep learning for the Twitter sentiment classification, considerable improvements in classification performance were observed (Wehrmann et al., 2017; Jianqiang et al., 2018; Naseem et al., 2020). Similarly to our approach, recent approaches use contextual embeddings such as ELMo (Peters et al., 2018) and BERT (Devlin et al., 2019), but in a monolingual setting.

\subsection{Transfer of trained models}

Cross-lingual word embeddings can be used directly as inputs in natural language processing models. The main idea is to train a model on data from one language and then apply it to another, relying on shared cross-lingual representation. Several tasks have been attempted in testing cross-lingual transfe. Søgaard et al. (2019) survey the transfer in the following tasks: document classification, dependency parsing, POS tagging, named entity recognition, super-sense tagging, semantic parsing, discourse parsing, dialogue state tracking, entity linking (wikification), sentiment analysis, machine translation, natural language interference, etc. For example, Ranasinghe 
and Zampieri (2020) apply large pretrained models in a similar way as we but use offensive language domain and only four languages from different families (English, Spanish, Bengali, and Hindu). In sentiment analysis, which is of particular interest in this work, Mogadala and Rettinger (2016) evaluate their embeddings on the multilingual Amazon product review dataset. In the Twitter sentiment analysis, Wehrmann et al. (2017) use LSTM networks but first learn a joint representation for four languages (English, German, Portuguese, and Spanish) with character-based convolutional neural networks.

\section{DATASETS AND EXPERIMENTAL SETTINGS}

This section presents the evaluation metrics, experimental data, and implementation details of the used neural prediction models.

\subsection{Evaluation metrics}

Following Mozetič et al. (2016), we report the $\bar{F}_{1}$ score and classification accuracy $(C A)$. The $F_{1}(c)$ score for class value $c$ is the harmonic mean of precision $p$ and recall $r$ for the given class $c$, where the precision is defined as the proportion of correctly classified instances from the instances predicted to be from the class $c$, and the recall is the proportion of correctly classified instances actually from the class $c$ :

$$
F_{1}(c)=\frac{2 p_{c} r_{c}}{p_{c}+r_{c}}
$$

The $F_{1}$ score returns values from the [0,1] interval, where 1 means perfect classification, and $\mathrm{o}$ indicates that either precision or recall for class $c$ is $\mathrm{o}$. We use an instance of the $F_{1}$ score specifically designed to evaluate the 3-class sentiment models (Kiritchenko et al., 2014). $\bar{F}_{1}$ is defined as the average over the positive (+) and negative ( - ) sentiment class:

$$
\bar{F}_{1}=\frac{F_{1}(+)+F_{1}(-)}{2} .
$$

$\bar{F}_{1}$ implicitly considers the ordering of sentiment values by considering only the extreme labels, positive (+) and negative (-). The middle, neutral, is taken 
into account indirectly. $\overline{F_{1}}=1$ implies that all negative and positive tweets were correctly classified, and as a consequence, all neutrals as well. $\bar{F}_{1}=\mathrm{o}$ indicates that all tweets were classified as neutral, and consequently, all negative and positive tweets were incorrectly classified.

$\bar{F}_{1}$ is not the best performance measure. First, taking the arithmetic average of the $F_{1}$ scores over different classes (called macro $F_{1}$ ) is methodologically misguided (Flach and Kull, 2015). It is justified only when the class distribution is approximately even, as in our case. Second, $\bar{F}_{1}$ does not account for correct classifications by chance. A more appropriate measure that allows for class ordering, classification by chance, and class labelling with disagreements is Krippendorff's alpha-reliability (Krippendorff, 2013). However, since $\bar{F}_{1}$ is commonly used in the sentiment classification community, and the results are typically well-correlated with the alpha-reliability, we decided to report our experimental results in terms of $\overline{F_{1}}$.

The second score we report is the classification accuracy CA, defined as the ratio of correctly predicted tweets $N_{c}$ to all the tweets $N$ :

$$
C A=\frac{N_{c}}{N}
$$

\subsection{Datasets}

We use a corpus of Twitter sentiment datasets (Mozetič et al., 2016), consisting of 15 languages, with over 1.6 million annotated tweets. The languages covered are Albanian, Bosnian, Bulgarian, Croatian, English, German, Hungarian, Polish, Portuguese, Russian, Serbian, Slovak, Slovene, Spanish, and Swedish. The authors studied the annotators' agreement on the labelled tweets. They discovered that the SVM classifier achieves significantly lower score for some languages (English, Russian, Slovak) than the annotators. This hints that there might be room for improvement for these languages using a better classification model or a larger training set.

We cleaned the above datasets by removing the duplicated tweets, weblinks, and hashtags. Due to the low quality of sentiment annotations indicated by low self-agreement and low inter-annotator agreement, we removed Albanian and Spanish datasets. For these two languages, the self-agreement expressed with $\bar{F}_{1}$ score is 0.60 and 0.49 , respectively; the inter-annotator agreement is 
0.41 and 0.42. As defined above, $\bar{F}_{1}$ is the arithmetic average of $F_{1}$ scores for the positive and negative tweets, where $F_{1}(c)$ is the fraction of equally labelled tweets out of all the tweets with the label $c$.

In the paper where the datasets were introduced (Mozetič et al., 2016), Serbian, Croatian, and Bosnian tweets were merged into a single dataset. The three languages are very similar and difficult to distinguish in short Twitter posts. However, it turned out that this merge resulted in a poor classification performance due to a very different quality of annotations. In particular, Serbian (71,721 tweets) was annotated by 11 annotators, where two of them accounted for over $40 \%$ of the annotations. All the inter-annotator agreement measures come from the Serbian only (1,880 tweets annotated twice by different annotators, $\bar{F}_{1}$ is 0.51$)$, and there are very few tweets annotated twice by the same annotator (182 tweets only, $\bar{F}_{1}$ for the self-agreement is o.46). In contrast, all the Croatian and Bosnian tweets were annotated by a single annotator, and we have reliable self-agreement estimates. There are 84,001 Croatian tweets, 13,290 annotated twice, and the self-agreement $\bar{F}_{1}$ is 0.83 . There are 38,105 Bosnian tweets, 6,519 annotated twice, and the self-agreement $\bar{F}_{1}$ is 0.78 . The authors concluded that the annotation quality of the Croatian and Bosnian tweets is considerably higher than that of the Serbian. If one constructs separate sentiment classifiers for each language, one observes a very different performance than reported originally. The individual classifiers are better and "well-behaved" compared to the joint Serbian/Croatian/Bosnian model. In this paper, we follow the authors' suggestion that datasets with no overlapping annotations and different annotation quality are better not merged. As a consequence, the Serbian, Croatian, and Bosnian datasets are analysed separately. The characteristics of all the 13 datasets are presented in Table 1. 
Table 1: The characteristics of datasets

\begin{tabular}{l|r|r|r|r|r|r} 
& \multicolumn{7}{c|}{ Number of tweets } & \multicolumn{2}{c}{ Agreement $\left(\overline{\boldsymbol{F}}_{1}\right)$} \\
\hline Language & Negative & Neutral & Positive & All & Self- & Inter- \\
\hline Bosnian & 12,868 & 11,526 & 13,711 & 38,105 & 0.78 & - \\
\hline Bulgarian & 15,140 & 31,214 & 20,815 & 67,169 & 0.77 & 0.50 \\
\hline Croatian & 21,068 & 19,039 & 43,894 & 84,001 & 0.83 & - \\
\hline English & 26,674 & 46,972 & 29,388 & 103,034 & 0.79 & 0.67 \\
\hline German & 20,617 & 60,061 & 28,452 & 109,130 & 0.73 & 0.42 \\
\hline Hungarian & 10,770 & 22,359 & 35,376 & 68,505 & 0.76 & - \\
\hline Polish & 67,083 & 60,486 & 96,005 & 223,574 & 0.84 & 0.67 \\
\hline Portuguese & 58,592 & 53,820 & 44,981 & 157,393 & 0.74 & - \\
\hline Russian & 34,252 & 44,044 & 29,477 & 107,773 & 0.82 & - \\
\hline Serbian & 24,860 & 30,700 & 16,161 & 71,721 & 0.46 & 0.51 \\
\hline Slovak & 18,716 & 14,917 & 36,792 & 70,425 & 0.77 & - \\
\hline Slovene & 38,975 & 60,679 & 34,281 & 133,935 & 0.73 & 0.54 \\
\hline Swedish & 25,319 & 17,857 & 15,371 & 58,547 & 0.76 & - \\
\hline
\end{tabular}

Note. The left-hand side reports the number of tweets from each category and the overall number of instances for individual languages. The right-hand side contains self-agreement of annotators and inter-annotator agreement for tried languages where more than one annotator was involved.

\subsection{Implementation details}

In our experiments, we use three different types of prediction models, BiLSTM neural networks using joint vector space embeddings constructed with the LASER library, and two variants of BERT, mBERT, and CSE BERT. The original mBERT (bert-multi-cased) is pretrained on 104 languages, has 12 transformer layers, and 110 million parameters. The CSE BERT uses the same architecture but is pretrained only on Croatian, Slovene, and English. In the construction of sentiment classification models, we fine-tune the whole network, using the batch size of 32, 2 epochs, and Adam optimiser. We also tested larger numbers of epochs and larger batch sizes in preliminary experiments, but this did not improve the performance.

The cross-lingual embeddings from the LASER library are pretrained on 93 languages, using BiLSTM networks, and are stored as 1024 dimensional embedding vectors. Our classification models contain an embedding layer, followed by a multilayer perceptron hidden layer of size 8 , and an output layer 
with three neurons (corresponding to three output classes, negative, neutral, and positive sentiment) using the softmax. We use the ReLU activation function and Adam optimiser. The fine-tuning uses a batch size of 32 and 10 epochs.

Further technical details are available in the freely available source code.

\section{EXPERIMENTS AND RESULTS}

Our experimental work focuses on model transfer with cross-lingual embeddings. However, to first establish the suitability of different embedding spaces for Twitter sentiment classification, we start with their comparison in a monolingual setting in Section 4.1. We compare the three neural approaches presented in Section 3.3 (common vector space of LASER, mBERT, and CSE BERT). As a baseline, we use the classical approach using bag-of-ngram representation with the SVM classifier. In the cross-lingual experiments, we focus on the two most-successful types of model transfer, described in Sections 2.2 and 2.3: the common vector space of the LASER library and the variants of the multilingual BERT model (mBERT and CSE BERT). We conducted several cross-lingual transfer experiments: transfer of models between languages from the same (Section 4.2) and different language family (Section 4.3), as well as the expansion of training sets with varying amounts of data from other languages (Section 4.4). In the experiments, we did not systematically test all possible combinations of languages and language groups as this would require an excessive amount of computational time and reporting space, and would not contribute to the clarity of the paper. Instead, we arbitrarily selected a representative set of language combinations in advance. We leave a comprehensive systematic approach based on informative features (Lin et al., 2019) for further work.

\subsection{Comparing embedding spaces}

To establish the appropriateness of different embedding approaches for our Twitter sentiment classification task, we start with experiments in a monolingual setting. We compare embeddings into a joint vector space obtained with the LASER library with mBERT and CSE BERT. Note that there is no transfer between different languages in this experiment but only a test of 
the suitability of the representation, i.e. embeddings. To make the results comparable with previous work on these datasets, we report results obtained with 10-fold blocked cross-validation. There is no randomisation of training examples in the blocked cross-validation, and each fold is a block of consecutive tweets. It turns out that standard cross-validation with a random selection of examples yields unrealistic estimates of classifier performance and should not be used to evaluate classifiers in time-ordered data scenarios (Mozetič et al., 2018).

As a baseline, we report the results of SVM models without neural embeddings that use Delta TF-IDF weighted bag-of-ngrams representation with substantial preprocessing of tweets (Mozetič et al., 2016). As the datasets for the Bosnian, Croatian, and Serbian languages were merged in (Mozetič et al., 2016) due to the similarity of these languages, we report the performance on the merged dataset for the SVM classifier. Results are presented in Table 2.

Table 2: Comparison of different representations: supervised mapping into a joint vector space with the LASER library, mBERT, CSE BERT, and bag-of-ngrams with the SVM classifier

\begin{tabular}{l|r|r|r|r|r|r|r|r} 
& \multicolumn{2}{c}{ LASER } & \multicolumn{2}{c|}{ mBERT } & \multicolumn{2}{c|}{ CSE BERT } & \multicolumn{2}{c}{ SVM } \\
\hline Language & $\overline{\boldsymbol{F}}_{1}$ & $\mathbf{C A}$ & $\overline{\boldsymbol{F}}_{\mathbf{1}}$ & $\mathbf{C A}$ & $\overline{\boldsymbol{F}}_{\mathbf{1}}$ & $\mathbf{C A}$ & $\overline{\boldsymbol{F}}_{\mathbf{1}}$ & CA \\
\hline Bosnian & $\mathbf{0 . 6 8}$ & 0.64 & 0.65 & 0.60 & $\mathbf{0 . 6 8}$ & $\mathbf{0 . 6 5}$ & $(0.61$ & $0.56)$ \\
\hline Bulgarian & 0.53 & $\mathbf{0 . 5 9}$ & $\mathbf{0 . 5 8}$ & $\mathbf{0 . 5 9}$ & 0.00 & 0.45 & 0.52 & 0.54 \\
\hline Croatian & 0.72 & 0.68 & 0.64 & 0.66 & $\mathbf{0 . 7 6}$ & $\mathbf{0 . 7 1}$ & $(0.61$ & $0.56)$ \\
\hline English & 0.62 & 0.65 & $\mathbf{0 . 6 8}$ & $\mathbf{0 . 6 8}$ & 0.67 & 0.66 & 0.63 & 0.64 \\
\hline German & 0.52 & 0.64 & $\mathbf{0 . 6 6}$ & $\mathbf{0 . 6 6}$ & 0.31 & 0.59 & 0.54 & 0.61 \\
\hline Hungarian & 0.63 & 0.67 & $\mathbf{0 . 6 5}$ & $\mathbf{0 . 6 9}$ & 0.57 & 0.65 & 0.64 & 0.67 \\
\hline Polish & $\mathbf{0 . 7 0}$ & 0.66 & $\mathbf{0 . 7 0}$ & $\mathbf{0 . 7 0}$ & 0.56 & 0.57 & 0.68 & 0.63 \\
\hline Portuguese & 0.48 & 0.47 & 0.50 & 0.49 & 0.12 & 0.22 & $\mathbf{0 . 5 5}$ & $\mathbf{0 . 5 1}$ \\
\hline Russian & $\mathbf{0 . 7 0}$ & $\mathbf{0 . 7 0}$ & 0.64 & 0.64 & 0.07 & 0.43 & 0.61 & 0.60 \\
\hline Serbian & 0.50 & 0.54 & 0.50 & 0.52 & 0.30 & 0.50 & $\mathbf{( 0 . 6 1}$ & $\mathbf{0 . 5 6 )}$ \\
\hline Slovak & $\mathbf{0 . 7 2}$ & $\mathbf{0 . 7 2}$ & 0.67 & 0.66 & 0.69 & 0.71 & 0.68 & 0.68 \\
\hline Slovene & 0.57 & 0.58 & 0.58 & 0.58 & $\mathbf{0 . 6 0}$ & $\mathbf{0 . 6 1}$ & 0.55 & 0.54 \\
\hline Swedish & $\mathbf{0 . 6 7}$ & 0.64 & $\mathbf{0 . 6 7}$ & $\mathbf{0 . 6 5}$ & 0.54 & 0.56 & 0.66 & 0.62 \\
\hline \#Best & 5 & 3 & 6 & 6 & 3 & 3 & 2 & 2 \\
\hline
\end{tabular}

Note. The best score for each language and metric is in bold. In the last row, we count the number of best scores for each model. The SVM results for Bosnian, Croatian, and Serbian were obtained with the model trained on the merged dataset of these languages model and are therefore not directly compatible with the language-specific results for the other representations. 
The SVM baseline using bag-of-ngrams representation mostly achieves lower predictive performance than the two neural embedding approaches. We speculate that the main reason is more information about the language structure contained in precomputed dense embeddings used by the neural approaches. Together with the fact that standard feature-based machine learning approaches require much more preprocessing effort, it seems that there are no good reasons why to bother with this approach in text classification; we, therefore, omit this method from further experiments. The mBERT model is the best of the tested methods, achieving the best $\bar{F}_{1}$ and CA scores in six languages (in bold), closely followed by the LASER approach, which achieves the best $\bar{F}_{1}$ score in five languages and the best CA score in three languages. The CSE BERT is specialised for only three languages, and it achieves the best scores in languages where it is trained (except in English, where it is close behind mBERT), and in Bosnian, which is similar to Croatian. Overall, it seems that large pretrained transformer models (mBERT and CSE BERT) are dominating in the Twitter sentiment prediction. The downside of these models is that their training, fine-tuning, and execution require more computational time than precomputed fixed embeddings. Nevertheless, with progress in optimisation techniques for neural network learning and advent of computationally more efficient BERT variants, e.g., (You et al., 2020), this obstacle might disappear in the future.

\subsection{Transfer to the same language family}

The transfer of prediction models between similar languages from the same language family is the most likely to be successful. We test several combinations of source and target languages from Slavic and Germanic language families. We report the results in Table 3.

In each experiment, we use the entire dataset(s) of the source language as the training set and the whole dataset of the target language as the testing set, i.e. we do a zero-shot transfer. We compare the results with the LASER embeddings with BiLSTM network using training and testing set from the target language, where $70 \%$ of the dataset is used for training and 30\% for testing. As we use large datasets, the latter results can be taken as an upper bound of what cross-lingual transfer models could achieve in ideal conditions. 
The results from Table 3 (bottom line) show that there is a gap in the performance of transfer learning models and native models. On average, the gap in $\bar{F}_{1}$ is $5 \%$ for the LASER approach, $6 \%$ for mBERT, and $8 \%$ for CSE BERT. For CA, the average gap is $7 \%$ for both LASER and mBERT and $8 \%$ for CSE BERT. However, there are significant differences between languages, and we advise to test both LASER and mBERT for a specific new language, as the models are highly competitive. The CSE BERT is slightly less successful measured with the average performance gap over all languages as the gap is $8 \%$ in both $\bar{F}_{1}$ and CA. However, if we take only the three languages used in the training of CSE BERT (Croatian, Slovene, and English) as shown in

Table 3: The transfer of trained models between languages from the same language family using LASER common vector space, mBERT, and CSE BERT

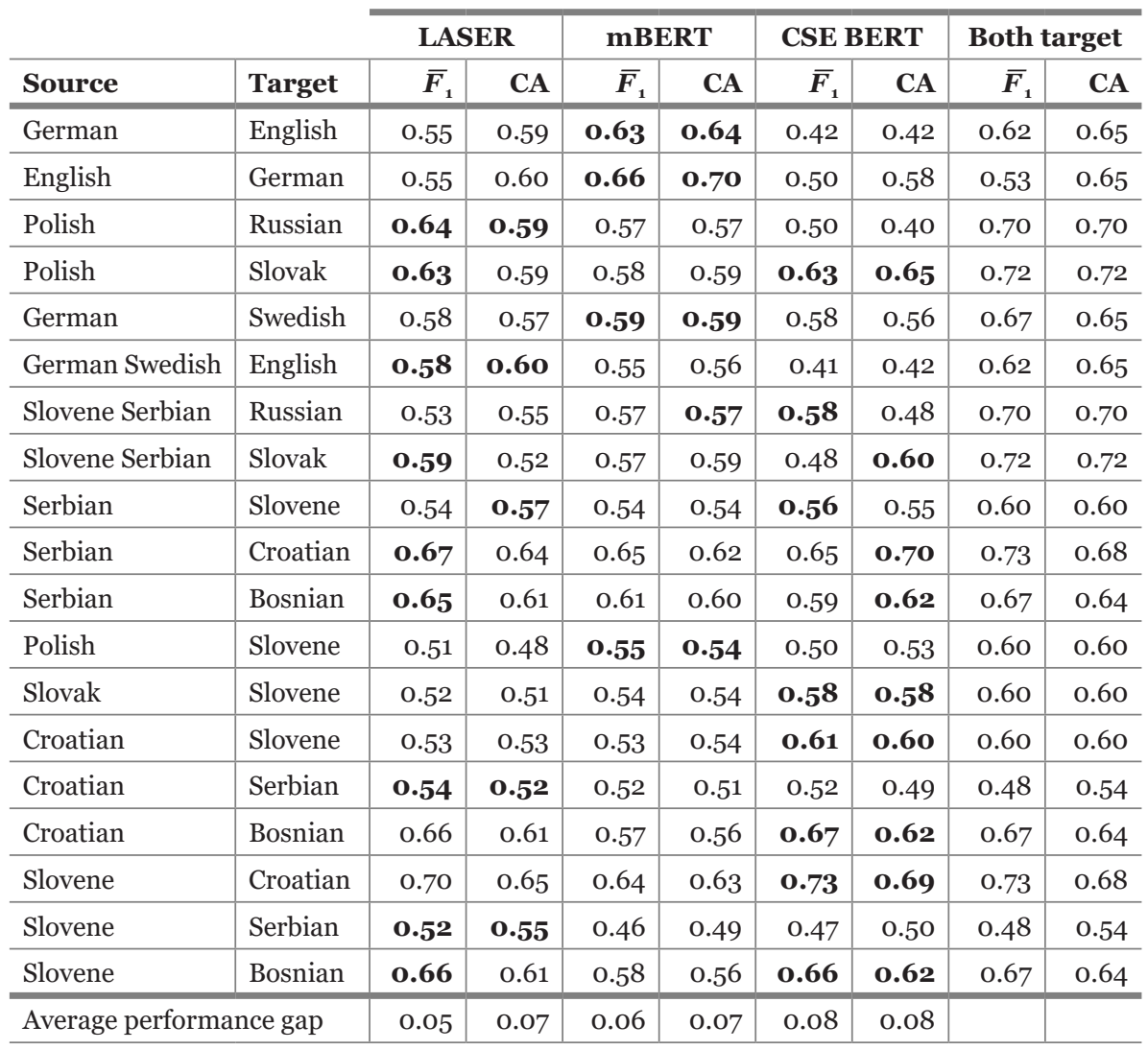

Note. We compare the results with both training and testing set from the target language using the LASER approach (the right-most two columns). 
Table 4, conclusions are entirely different. The average performance gap is $0 \%$ in $\bar{F}_{1}$ and $1 \%$ in the classification accuracy, meaning that we get almost a perfect cross-lingual transfer for these languages on the Twitter sentiment prediction task.

We also tried more than one input language at once, for example, German and Swedish as source languages and English as the target language, as shown in Table 3. The success of the tested combinations is mixed: for some models and some languages, we slightly improve the scores, while for others, we slightly decrease them. We hypothesise that our datasets for individual languages are large enough so that adding additional training data does not help.

Table 4: The transfer of sentiment models between all combinations of languages on which CSE BERT was trained (Croatian, Slovene, and English)

\begin{tabular}{l|l|r|r|r|r|r|r|r|r}
\multicolumn{1}{c}{} & \multicolumn{2}{c}{ LASER } & \multicolumn{2}{c|}{ mBERT } & \multicolumn{2}{c}{ CSE BERT } & \multicolumn{2}{c}{ Both target } \\
\hline Source & Target & $\overline{\boldsymbol{F}}_{\mathbf{1}}$ & $\mathbf{C A}$ & $\overline{\boldsymbol{F}}_{\mathbf{1}}$ & $\mathbf{C A}$ & $\overline{\boldsymbol{F}}_{\mathbf{1}}$ & $\mathbf{C A}$ & $\overline{\boldsymbol{F}}_{\mathbf{1}}$ & $\mathbf{C A}$ \\
\hline Croatian & Slovene & 0.53 & 0.53 & 0.53 & 0.54 & $\mathbf{0 . 6 1}$ & $\mathbf{0 . 6 0}$ & 0.60 & 0.60 \\
\hline Croatian & English & $\mathbf{0 . 6 3}$ & 0.63 & $\mathbf{0 . 6 3}$ & $\mathbf{0 . 6 6}$ & 0.62 & 0.64 & 0.62 & 0.65 \\
\hline English & Slovene & 0.54 & 0.57 & 0.50 & 0.53 & $\mathbf{0 . 5 9}$ & $\mathbf{0 . 5 7}$ & 0.60 & 0.60 \\
\hline English & Croatian & 0.62 & $\mathbf{0 . 6 7}$ & 0.67 & 0.63 & $\mathbf{0 . 7 3}$ & $\mathbf{0 . 6 7}$ & 0.73 & 0.68 \\
\hline Slovene & English & 0.63 & 0.64 & $\mathbf{0 . 6 5}$ & $\mathbf{0 . 6 7}$ & 0.63 & 0.64 & 0.62 & 0.65 \\
\hline Slovene & Croatian & 0.70 & 0.65 & 0.64 & 0.63 & $\mathbf{0 . 7 3}$ & $\mathbf{0 . 6 9}$ & 0.73 & 0.68 \\
\hline Croatian English & Slovene & 0.54 & 0.54 & 0.53 & 0.54 & $\mathbf{0 . 6 0}$ & $\mathbf{0 . 5 8}$ & 0.60 & 0.60 \\
\hline Croatian Slovene & English & 0.62 & 0.61 & $\mathbf{0 . 6 5}$ & $\mathbf{0 . 6 7}$ & 0.63 & 0.65 & 0.62 & 0.65 \\
\hline English Slovene & Croatian & 0.64 & 0.68 & 0.63 & 0.63 & $\mathbf{0 . 6 8}$ & $\mathbf{0 . 7 0}$ & 0.73 & 0.68 \\
\hline
\end{tabular}

\subsection{Transfer to a different language family}

The transfer of prediction models between languages from different language families is less likely to be successful. Nevertheless, to observe the difference, we test several combinations of source and target languages from different language families (one from Slavic, the other from Germanic, and vice-versa). We compare the LASER approach with mBERT models; the CSE BERT is not constructed for this setting, and we skip it in this experiment. We report the results in Table 5 . 
The results show that with the LASER approach, there is an average decrease of performance for transfer learning models of $11 \%$ (both $\bar{F}_{1}$ and CA), and for mBERT, the gap is $9 \%$. This gap is significant and makes the resulting transferred models less useful in the target languages, though there are considerable differences between the languages.

Table 5: The transfer of trained models between languages from different language families using LASER common vector space and mBERT

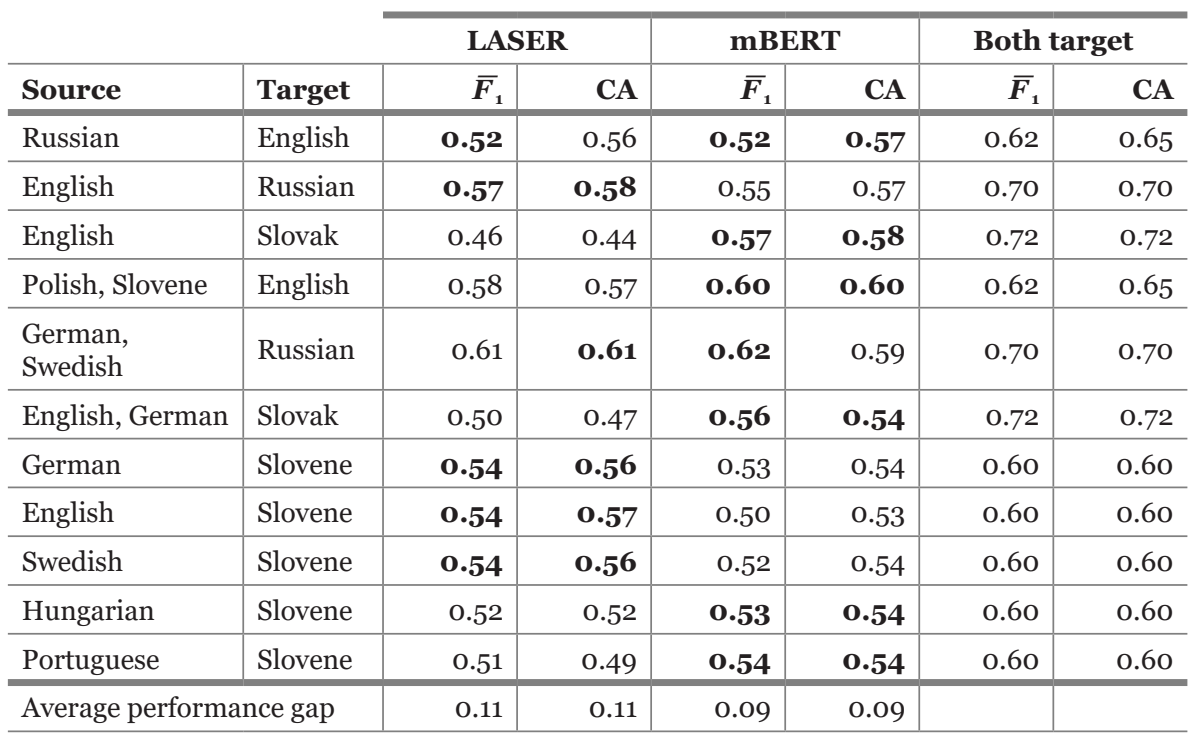

Note. We compare the results with both training and testing set from the target language using the LASER approach (the right-most two columns).

\subsection{Increasing datasets with several languages}

Another type of cross-lingual transfer is possible if we increase the training sets with instances from several related and unrelated languages. We conduct two sets of experiments in this scenario. In the first setting, reported in Table 6, we constructed the training set in each experiment with instances from several languages and $70 \%$ of the target language dataset. The remaining $30 \%$ of target language instances are used as the testing set. In the second setting, reported in Table 7, we merge all other languages and 70\% of the target language into a joint training set. We compare the LASER approach, mBERT, and also CSE BERT, as Slovene and Croatian are involved in some combinations. 
Table 6 shows a gap between learning models using the expanded datasets and models with only target language data. The decrease is more extensive for both BERT models (on average around 10\%) than for the LASER approach (the decrease is on average $3 \%$ for $\overline{\boldsymbol{F}}_{1}$ and $5 \%$ for $\mathrm{CA}$ ). These results indicate that the tested expansion of datasets was unsuccessful, i.e. the provided amount of training instances in the target language was already sufficient for successful learning. The additional instances from other languages in the transformed space are likely to be of lower quality than the native instances and therefore decrease the performance.

Table 6: The expansion of training sets with instances from several languages

\begin{tabular}{l|l|r|r|r|r|r|r|r|r}
\multicolumn{1}{c}{} & \multicolumn{2}{c|}{ LASER } & \multicolumn{2}{c|}{ mBERT } & \multicolumn{2}{c|}{ CSEBERT } & \multicolumn{2}{c}{ Target only } \\
\hline Source & Target & $\overline{\boldsymbol{F}}_{\mathbf{1}}$ & $\mathbf{C A}$ & $\overline{\boldsymbol{F}}_{\mathbf{1}}$ & $\mathbf{C A}$ & $\overline{\boldsymbol{F}}_{\mathbf{1}}$ & $\mathbf{C A}$ & $\overline{\boldsymbol{F}}_{\mathbf{1}}$ & $\mathbf{C A}$ \\
\hline $\begin{array}{l}\text { English, Croatian, } \\
\text { Slovene }\end{array}$ & Slovene & 0.58 & 0.53 & 0.46 & 0.45 & $\mathbf{0 . 6 0}$ & $\mathbf{0 . 5 8}$ & 0.60 & 0.60 \\
\hline $\begin{array}{l}\text { English, Croatian, } \\
\text { Serbian, Slovak }\end{array}$ & Slovak & $\mathbf{0 . 6 7}$ & $\mathbf{0 . 6 5}$ & 0.57 & 0.54 & 0.27 & 0.37 & 0.72 & 0.72 \\
\hline $\begin{array}{l}\text { Hungarian, } \\
\text { Slovak, English, } \\
\text { Croatian, Russian }\end{array}$ & Russian & $\mathbf{0 . 6 7}$ & $\mathbf{0 . 6 5}$ & 0.61 & 0.59 & 0.63 & 0.61 & 0.70 & 0.70 \\
\hline $\begin{array}{l}\text { Russian, Swedish, } \\
\text { English }\end{array}$ & English & 0.60 & 0.61 & $\mathbf{0 . 6 2}$ & 0.60 & 0.59 & $\mathbf{0 . 6 2}$ & 0.62 & 0.65 \\
\hline $\begin{array}{l}\text { Croatian, Serbian, } \\
\text { Bosnian, Slovene }\end{array}$ & Slovene & 0.54 & $\mathbf{0 . 5 8}$ & 0.44 & 0.45 & $\mathbf{0 . 5 7}$ & 0.56 & 0.60 & 0.60 \\
\hline $\begin{array}{l}\text { English, Swedish, } \\
\text { German }\end{array}$ & German & 0.55 & 0.60 & $\mathbf{0 . 6 0}$ & $\mathbf{0 . 6 4}$ & 0.47 & 0.58 & 0.53 & 0.65 \\
\hline $\begin{array}{l}\text { Average } \\
\text { performance gap }\end{array}$ & & 0.03 & 0.05 & 0.08 & 0.11 & 0.11 & 0.10 & & \\
\hline
\end{tabular}

Note. We compare the LASER approach, mBERT, and CSE BERT. As the upper bound, we give results of the LASER approach trained on only the target language.

The results in Table 7, where we test the expansion of the training set (consisting of $70 \%$ of the dataset in the target language) with all other languages, show that using many languages and significant enlargement of datasets is also not successful. The two improvements in the LASER approach over using only target language are limited to a single metric ( $F_{1}$ in case of Bulgarian and Serbian), which indicates that true positives are favoured at the expense of true negatives. For all the other languages, the tried expansions of training sets are unsuccessful for the LASER approach; the difference to native models 
is on average $3.5 \%$ for the $\bar{F}_{1}$ score and $6 \%$ for CA. The mBERT models are in almost all cases more successful in this massive transfer than LASER models, and they sometimes marginally beat the reference mBERT approach trained only on the target language.

Table 7: The expansion of training sets with instances from all other languages $(+70 \%$ of the target language instances) to train the LASER approach and mBERT

\begin{tabular}{l|r|r|r|r|r|r|r|r} 
& \multicolumn{4}{c}{ LASER } & \multicolumn{4}{c}{ mBERT } \\
\cline { 2 - 12 } & \multicolumn{1}{c}{ All \& Target } & \multicolumn{1}{c|}{ Only Target } & \multicolumn{2}{c}{ All \&Target } & \multicolumn{2}{c}{ Only Target } \\
\hline Target & $\overline{\boldsymbol{F}}_{\mathbf{1}}$ & $\mathbf{C A}$ & $\overline{\boldsymbol{F}}_{\mathbf{1}}$ & $\mathbf{C A}$ & $\overline{\boldsymbol{F}}_{\mathbf{1}}$ & $\mathbf{C A}$ & $\overline{\boldsymbol{F}}_{\mathbf{1}}$ & $\mathbf{C A}$ \\
\hline Bosnian & 0.64 & 0.59 & 0.67 & 0.64 & 0.63 & 0.60 & 0.65 & 0.60 \\
\hline Bulgarian & $\mathbf{0 . 5 4}$ & 0.56 & 0.50 & 0.59 & $\mathbf{0 . 6 0}$ & $\mathbf{0 . 6 0}$ & 0.58 & 0.59 \\
\hline Croatian & 0.63 & 0.57 & 0.73 & 0.68 & $\mathbf{0 . 6 5}$ & 0.63 & 0.64 & 0.66 \\
\hline English & 0.58 & 0.60 & 0.62 & 0.65 & 0.64 & $\mathbf{0 . 6 9}$ & 0.68 & 0.68 \\
\hline German & 0.52 & 0.59 & 0.53 & 0.65 & 0.61 & 0.66 & 0.66 & 0.66 \\
\hline Hungarian & 0.59 & 0.61 & 0.60 & 0.67 & 0.65 & 0.69 & 0.65 & 0.69 \\
\hline Polish & 0.67 & 0.63 & 0.70 & 0.66 & $\mathbf{0 . 7 1}$ & $\mathbf{0 . 7 1}$ & 0.70 & 0.70 \\
\hline Portuguese & 0.44 & 0.39 & 0.52 & 0.51 & $\mathbf{0 . 5 2}$ & $\mathbf{0 . 5 2}$ & 0.50 & 0.49 \\
\hline Russian & 0.66 & 0.64 & 0.70 & 0.70 & $\mathbf{0 . 6 7}$ & $\mathbf{0 . 6 6}$ & 0.64 & 0.64 \\
\hline Serbian & $\mathbf{0 . 5 2}$ & 0.49 & 0.48 & 0.54 & $\mathbf{0 . 5 3}$ & 0.51 & 0.50 & 0.52 \\
\hline Slovak & 0.64 & 0.61 & 0.72 & 0.72 & 0.67 & 0.65 & 0.67 & 0.66 \\
\hline Slovene & 0.54 & 0.50 & 0.60 & 0.60 & 0.56 & 0.54 & 0.58 & 0.58 \\
\hline Swedish & 0.63 & 0.59 & 0.67 & 0.65 & 0.67 & 0.64 & 0.67 & 0.65 \\
\hline Avg. gap & 0.03 & 0.06 & & & 0.00 & 0.00 & & \\
\hline
\end{tabular}

Note. We compare the results with the training on only the target language. The scores where models with the expanded training sets beat their respective reference scores are in bold.

\section{CONCLUSIONS}

We studied state-of-the-art approaches to the cross-lingual transfer of Twitter sentiment prediction models: mappings of words into the common vector space using the LASER library and two multilingual BERT variants (mBERT and trilingual CSE BERT). Our empirical evaluation is based on relatively large datasets of labelled tweets from 13 European languages. We first tested the success of these text representations in a monolingual setting. The results show that BERT variants are the most successful, closely followed by the LASER approach, while the classical bag-of-ngrams coupled with the SVM 
classifier is no longer competitive with neural approaches. In the cross-lingual experiments, the results show that there is a significant transfer potential using the models trained on similar languages; compared to training and testing on the same language, with LASER, we get on average $5 \%$ lower $\overline{F_{1}}$ score and with mBERT $6 \%$ lower $\bar{F}_{1}$ score. The transfer of models with CSE BERT is even more successful in the three languages covered by this model, where we get no performance gap compared to the LASER approach trained and tested on the target language. Using models trained on languages from different language families produces larger differences (on average around 10\% for $\bar{F}_{1}$ and CA). Our attempt to expand training sets with instances from different languages was unsuccessful using either additional instances from a small group of languages or instances from all other languages. The source code of our analyses is freely available ${ }^{3}$.

We plan to expand BERT models with additional emotional and subjectivity information in future work on sentiment classification. Given the favourable results in cross-lingual transfer, we will expand the work to other relevant tasks.

\section{Acknowledgments}

The research was supported by the Slovene Research Agency through research core funding no. P6-0411 and P2-103, as well as project no. J6-2581. This paper is supported by European Union's Horizon 2020 Programme project EMBEDDIA (Cross-Lingual Embeddings for Less-Represented Languages in European News Media, grant no. 825153), and Rights, Equality and Citizenship Programme project IMSyPP (Innovative Monitoring Systems and Prevention Policies of Online Hate Speech, grant no. 875263). The results of this publication reflect only the authors' view, and the Commission is not responsible for any use that may be made of the information it contains. 


\section{REFERENCES}

Artetxe, M., Labaka, G., \& Agirre, E. (2018a). Generalising and improving bilingual word embedding mappings with a multi-step framework of linear transformations. In Thirty-Second AAAI Conference on Artificial Intelligence.

Artetxe, M., Labaka, G., \& Agirre, E. (2018b). A robust self-learning method for fully unsupervised crosslingual mappings of word embeddings. In Proceedings of the 56th Annual Meeting of the Association for Computational Linguistics:Vol 1 (Long Papers) (pp. 789-798).

Artetxe, M., \& Schwenk, H. (2019). Massively multilingual sentence embeddings for zero-shot crosslingual transfer and beyond. Transactions of the Association for Computational Linguistics, 7, 597-610.

Bojanowski, P., Grave, E., Joulin, A., \& Mikolov, T. (2017). Enriching word vectors with subword information. Transactions of the Association for Computational Linguistics, 5, 135-146.

Conneau, A., Lample, G., Ranzato, M.A., Denoyer, L., \& J'egou, H. (2018). Word' translation without parallel data. In 6th Proceedings of International Conference on Learning Representation (ICLR). Retrieved from https://openreview.net/pdf?id=H196sainb

Devlin, J., Chang, M.-W., Lee, K., \& Toutanova, K. (2019). BERT: Pre-training of deep bidirectional transformers for language understanding. In Proceedings of the 2019 Conference of the North American Chapter of the Association for Computational Linguistics: Human Language Technologies, Vol. 1 (Long and Short Papers) (pp. 4171-4186).

Flach, P., \& Kull, M. (2015). Precision-recall-gain curves: PR analysis done right. In Advances in Neural Information Processing Systems (NIPS) (pp. 838-846).

Jianqiang, Z., Xiaolin, G., and Xuejun, Z. (2018). Deep convolution neural networks for Twitter sentiment analysis. IEEE Access, 6, 23253-23260.

Kiritchenko, S., Zhu, X., Mohammad, S. M. (2014). Sentiment analysis of short informal texts. Journal of Artificial Intelligence Research, 5o, 723-762.

Krippendorff, K. (2013). Content Analysis, An Introduction to Its Methodology (3rd ed.) Thousand Oaks, CA, USA: Sage Publications. 
Lin, Y. H., Chen, C. Y., Lee, J., Li, Z., Zhang, Y., Xia, M., Rijhwani, S., et al. (2019). Choosing transfer languages for cross-lingual learning. In Proceedings of the 57th Annual Meeting of the Association for Computational Linguistics (ACL) (pp. 3125-3135).

Mikolov, T., Le, Q. V., \& Sutskever, I. (2013). Exploiting similarities among languages for machine translation. arXiv preprint 1309.4168.

Mogadala, A., \& Rettinger, A. (2016). Bilingual word embeddings from parallel and non-parallel corpora for cross-language text classification. In Proceedings of NAACL-HLT (pp. 692-702).

Mozetič, I., Grčar, M., \& Smailović, J. (2016). Multilingual Twitter sentiment classification: The role of human annotators. PLOS ONE, 11(5). doi: 10.1371/ journal.pone. 0155036

Mozetič, I., Torgo, L., Cerqueira, V., \& Smailović, J. (2018). How to evaluate sentiment classifiers for Twitter time-ordered data? PLoS ONE 13(3).

Naseem, U., Razzak, I., Musial, K., \& Imran, M. (2020). Transformer based deep intelligent contextual embedding for Twitter sentiment analysis. Future Generation Computer Systems, 113, 58-69.

Peters, M., Neumann, M., Iyyer, M., Gardner, M., Clark, C., Lee, K., \& Zettlemoyer, L. (2018). Deep contextualised word representations. In Proceedings of the 2018 Conference of the North American Chapter of the Association for Computational Linguistics: Human Language Technologies, Vol. 1 (Long Papers) (pp. 2227-2237).

Ranasinghe, T., \& Zampieri, M. (2020). Multilingual Offensive Language Identification with Cross-lingual Embeddings. In Proceedings of the 2020 Conference on Empirical Methods in Natural Language Processing (EMNLP) (pp. 5838-5844).

Rosenthal, S., Nakov, P., Kiritchenko, S., Mohammad, S. M., Ritter, A., \& Stoyanov, V. (2015). SemEval-2015 task 10: Sentiment Analysis in Twitter. In Proceedings of 9th International Workshop on Semantic Evaluation (SemEval) (pp. 451-463).

Saif, H., Fernández, M., He, Y., Alani, H.(2013). Evaluation datasets for Twitter sentiment analysis: A survey and a new dataset, the STS-Gold. In 1st Intl. Workshop on Emotion and Sentiment in Social and Expressive Media: Approaches and Perspectives from AI (ESSEM). 
Søgaard, A., Vulić, I., Ruder, S., \& Faruqui, M. (2019). Cross-Lingual Word Embeddings. Morgan \& Claypool Publishers.

Ulčar, M., \& Robnik-Šikonja, M. (2020). FinEst BERT and CroSloEngual BERT. In International Conference on Text, Speech, and Dialogue (TSD) (pp. 104-111).

Vaswani, A., Shazeer, N., Parmar, N., Uszkoreit, J., Jones, L., Gomez, A. N., Kaiser, Ł., \& Polosukhin, I. (2017). Attention is all you need. In Advances in Neural Information Processing Systems (NIPS) (pp. 5998-60o8).

Virtanen, A., Kanerva, J., Ilo, R., Luoma, J., Luoto-lahti, J., Salakoski, T., Ginter, F., \& Pyysalo, S. (2019). Multilingual is not enough: BERT for Finnish. arXiv preprint 1912.07076.

Wehrmann, J., Becker, W., Cagnini, H. E., \& Barros, R. C. (2017). A character-based convolutional neural network for language-agnostic Twitter sentiment analysis. In 2017 International Joint Conference on Neural Networks (IJCNN) (pp. 2384-2391).

You, Y., Li, J., Reddi, S., Hseu, J., Kumar, S., Bhojanapalli, S., Song, X., et al. (2020). Large batch optimization for deep learning: Training BERT in 76 minutes. In 8th International Conference on Learning Representations (ICLR), 26-3o April, 202O, Addis Ababa, Ethiopia. 


\section{MEDJEZIKOVNI PRENOS KLASIFIKATORJEV SENTIMENTA}

Vektorske vložitve predstavijo besede v številski obliki tako, da so semantične relacije med besedami zapisane kot razdalje in smeri v vektorskem prostoru. Medjezikovne vložitve poravnajo vektorske prostore različnih jezikov, kar podobne besede v različnih jezikih postavi blizu skupaj. Medjezikovna poravnava lahko deluje na parih jezikov ali s konstrukcijo skupnega vektorskega prostora več jezikov. Medjezikovne vektorske vložitve lahko uporabimo za prenos modelov strojnega učenja med jeziki in s tem razrešimo težavo premajhnih ali neobstoječih učnih množic v jezikih z manj viri. V delu uporabljamo medjezikovne vložitve za prenos napovednih modelov strojnega učenja za napovedovanje sentimenta tvitov med trinajstimi jeziki. Osredotočeni smo na dva, v zadnjem času najuspešnejša, načina prenosa modelov. Prvi način uporablja modele naučene na skupnem vektorskem prostoru za mnoge jezike, izdelanem s knjižnico LASER. Drugi način uporablja velike, na mnogih jezikih vnaprej naučene, jezikovne modele tipa BERT. Naši poskusi kažejo, da je prenos modelov med podobnimi jeziki smiseln tudi povsem brez učnih podatkov v ciljnem jeziku. Uspešnost večjezikovnih modelov BERT in LASER je primerljiva, razlike so odvisne od jezika. Medjezikovni prenos z modelom CroSloEngual BERT, predhodno naučenim na le treh jezikih, je v teh in nekaterih sorodnih jezikih še precej boljši.

Ključne besede: obdelava naravnega jezika, strojno učenje, vektorske vložitve besedil, analiza sentimenta, modeli BERT

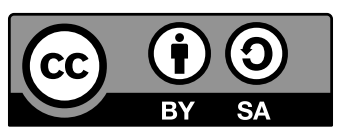

To delo je ponujeno pod licenco Creative Commons: Priznanje avtorstva-Deljenje pod enakimi pogoji 4.o Mednarodna. / This work is licensed under the Creative Commons Attribution-ShareAlike 4.o International. 